\title{
MEJORA DE LA PERFORMANCE DEL ACERO 1040 PARA LA FABRICACIÓN DE BARRAS DE CONSTRUCCIÓN*
}

\section{RESUMEN}

William Sergio Flores Roman ${ }^{1}$

Los aceros para armaduras de hormigón tienen que exhibir una buena relación entre resistencia y tenacidad que se ve reflejado en la performance del producto en servicio al ser aplicado a esfuerzos exigidos. Acerbrag es una empresa que produce aceros para la industria de la construcción teniendo en su gama los aceros de medio carbono para la fabricación de barras conformadas de dureza natural para armaduras en estructuras de hormigón. El objetivo de este trabajo es encontrar criterios de ventanas de trabajos operacionales y de control para la manufactura de aceros con buenas propiedades mecánicas en términos de resistencia y tenacidad. Este paper muestra los resultados de los trabajos realizados en las diferentes áreas productivas para conseguir un producto final con mayor resistencia a altas exigencias en servicio, además, reporta diferentes aspectos del proceso de manufactura del producto que van desde el ajuste de la composición química del acero, incluyendo criterios de propiedades mecánicas objetivos, criterios de calidad interna mostrando la microestructura de la barra antes y después de los cambios y finalmente la calidad dimensional y superficial de la barra nervurada, este último potenciando la falla prematura del material. Todos estos cambios, se ven reflejados finalmente en la performance de la barra, sometida a los nuevos criterios de exigencia en los ensayos mecánicos.

Palabras-claves: aceros de medio carbono; elementos residuales; propiedades mecánicas; ensayo de doblado

\section{IMPROVEMENT OF THE PERFORMANCE OF STEEL GRADE 1040 FOR THE MANUFACTURE OF REINFORCING BAR}

\begin{abstract}
Concrete reinforcing steels have to exhibit a good relationship between strength and toughness which is reflected by the performance of the product in service when applied to the required stresses. Acerbrag is a company that produces steels for the construction industry, having in its range medium carbon steels for the manufacture of shaped bars of natural hardness for reinforcement in concrete structures. The objective of this work is to understand the variables that make the material show good mechanical properties in terms of strength and toughness, as well as understanding the surface conditions that cause the material to fail. This paper shows the results of the work carried out in different production areas in order to obtain a final product with higher service requirement. This paper will report different aspects of the manufacturing process of the product, such as criterium for adjustment of the chemical composition of the steel, also includes criteria of mechanical properties objective, internal quality criteria showing the microstructure of the bar before and after the changes, finally dimensional and superficial quality of the bar. All these changes are finally reflected together with the performance of the bar with new severe testing methods.
\end{abstract}

Key words: medium carbon steel; residual elements, mechanical properties, bending test.

1 Mmet, MPhil, Beng en Metalurgia, University of Sheffield, Inglaterra, Jefe de Calidad de AcerBrag-Votorantim , Argentina. 


\section{INTRODUCCIÓN}

Las barras nervuradas para la industria de la construcción en Argentina cumplen la Norma IRAM/IAS U-500-528 barras conformadas de dureza natural para armaduras en estructuras de hormigón [1].Los nuevos desafíos de competitividad en costo de producción ligado directamente con el uso de insumos y materias primas de bajo costo, ocasionan cambios en la calidad del producto pudiendo llevar a reclamos de clientes, además de una percepción negativa en el mercado. La norma IRAM/IAS U-500-528 contempla una serie de requisitos como propiedades mecánicas, requisitos dimensionales y condiciones de ensayo de doblado tabla 1. Basados en las expectativas de calidad solicitadas por el cliente, el cual muchas veces exige una calidad de acero cuyas propiedades son superiores a las exigidas por la norma Figura 1, se realizó este trabajo de mejora de las barras nervuradas de acero de medio carbón mostrando una mayor tenacidad y resistencia a esfuerzos exigidos sin perder el foco en el costo.

Tabla 1 Propiedades de barras nervuradas especificadas en IRAM/IAS U500-528

\begin{tabular}{|c|c|c|c|c|c|}
\hline \multicolumn{3}{|c|}{ Ensayo de Doblado } & \multicolumn{3}{|c|}{ Ensayo de tracción ADN 420} \\
\hline $\begin{array}{c}\varnothing ́ \text { Barra } \\
\text { Nervurada }\end{array}$ & $\begin{array}{c}\varnothing \text { Mandril } \\
(\mathrm{mm})\end{array}$ & $\begin{array}{c}\text { Distancia } \\
\text { entre apoyos } \\
(\mathrm{mm})\end{array}$ & $\begin{array}{l}\text { Límite de } \\
\text { Fluencia } \\
\text { (MPa) (min) }\end{array}$ & $\begin{array}{l}\text { Resistencia } \\
\text { a la tracción } \\
\text { (MPa) (min) }\end{array}$ & $\begin{array}{c}\% \\
\text { Alargamiento }\end{array}$ \\
\hline 6 & 21 & 39 & \multirow{3}{*}{420} & \multirow{3}{*}{500} & \multirow{3}{*}{12} \\
\hline 8 & 28 & 52 & & & \\
\hline 10 & 35 & 65 & & & \\
\hline
\end{tabular}
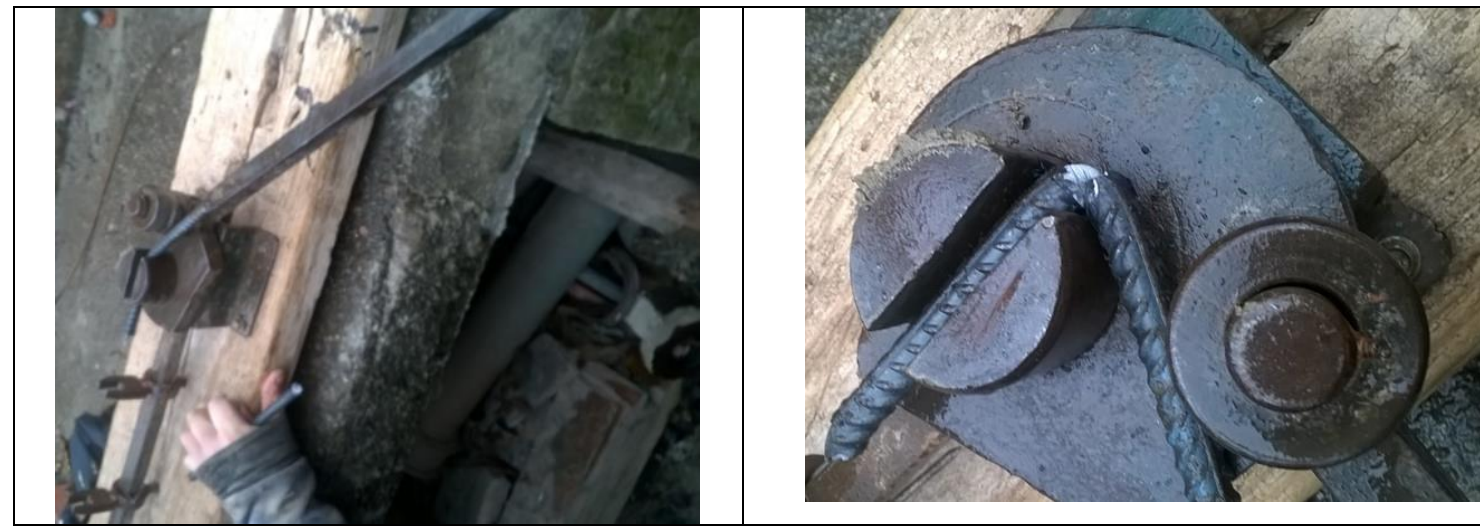

Figura 1. Muestra el ensayo de doblado de una barra de ADN $10 \mathrm{~mm}$ acero 1040

\subsection{DATOS ESTADISTICOS DE LA PERFORMANCE DE LA BARRA EN EL ENSAYO DE DOBLADO}

Uno de los problemas de las barras nervuradas es la resistencia del material cuando es sobre exigido al ensayo de doblado. Esta fractura prematura del material ocasiona reclamos de clientes incluso cuando las barras cumplen la norma. 
Se realizó pruebas de exigencia de doblado en las barras nervuradas $10 \mathrm{~mm}$ para analizar la capacidad de respuesta del material a ensayos exigentes. La Figura 2 muestra los ensayos de plegado a diferentes mandriles donde se muestra ensayos con mandril según norma con diámetro $35 \mathrm{~mm}$, seguidamente a un mandril más exigente con diámetro $18 \mathrm{~mm}$ y finalmente a una sobre exigencia mayor, con un mandril de diámetro $11 \mathrm{~mm}$. Como se aprecia, la performance del material cambia aumentando las roturas, grietas y fisuras cuando mayor es la severidad del ensayo.

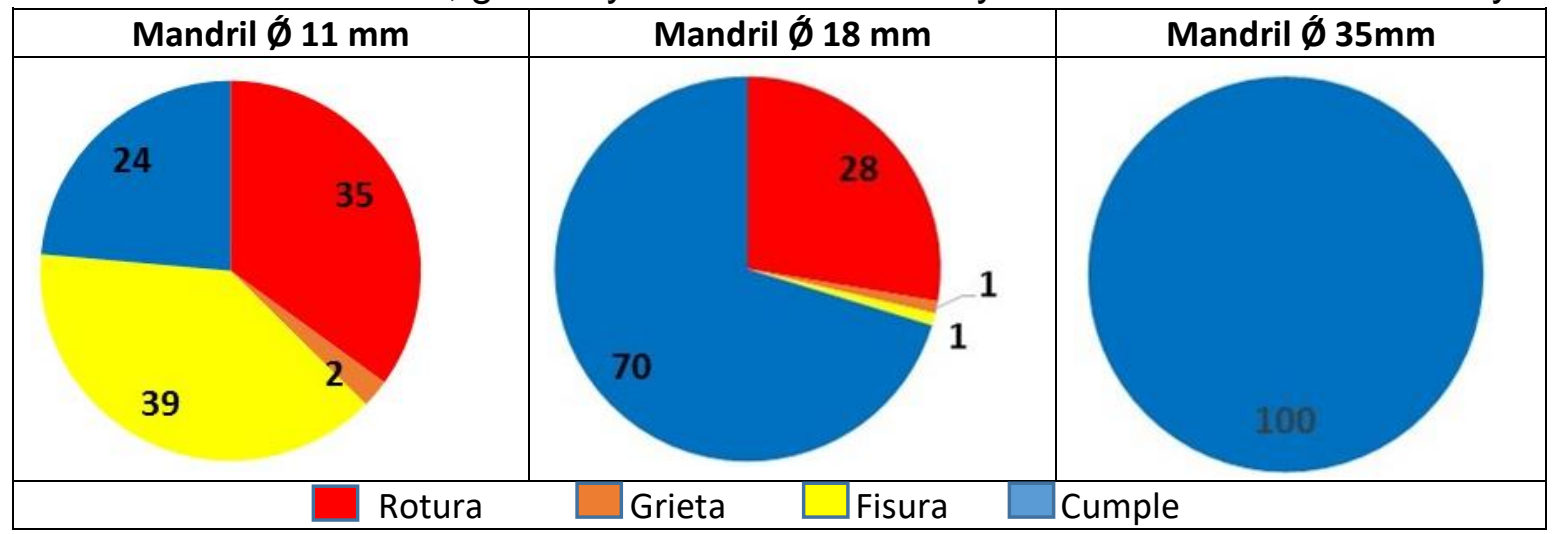

Figura 2. Porcentaje de material ensayado a diferentes mandriles de acuerdo a norma y a mandril exigente, barra nervurada $10 \mathrm{~mm}$ acero de calidad 1040. (Cantidad de muestras ensayadas 271).

\section{MATERIALES Y METODOS}

Los materiales utilizados en este estudio son barras de calidad de acero 1040 de diámetro $10 \mathrm{~mm}$. Estas barras son ensayadas con los siguientes equipos:

- Máquina de tracción INSTRON de capacidad 1000 KN con una velocidad de deformación de $100 \mathrm{~mm} / \mathrm{min}$

- Maquina dobladora CIFIC con mandriles intercambiables $11 \mathrm{~mm}, 16 \mathrm{~mm}$, $18 \mathrm{~mm}, 21 \mathrm{~mm}, 28 \mathrm{~mm}$ y $35 \mathrm{~mm}$.

- Microscopio óptico metalográfico para estudiar la microestructura de los diferentes cambios realizados en el proceso.

- Software procesador de datos Minitab para análisis estadístico y análisis de regresión.

\section{RESULTADOS Y DISCUSIÓN}

\subsection{ANÁLISIS DE FALLA, CARACTERIZACIÓN DE LA FRACTURA.}

La Figura 3 muestra los tipos de fractura que van cambiando de apariencia a medida que va perdiendo ductibilidad, desde un material muy dúctil mostrando una deformación grosera Caso 1 (fractura fibrosa) hasta un caso de un material completamente frágil de baja deformación con una falla de rápida fractura Caso 4 . La Figura 4 muestra el ángulo de corte de un material dúctil y material frágil, donde éste último muestra una dirección de fractura perpendicular a la tangente donde se concentra la máxima tensión. La Figura 5 muestra las metalografías de los 
materiales estudiados en la Figura 3 donde se muestra una disminución de la fase ferrita a medida que aumenta el área frágil.

Uno de las principales partes de este paper es determinar las principales causas que producen esta falla prematura del material y mostrar escenarios de modelamiento y control para consistencia de resultados de propiedades mecánicas a lo largo de la producción continua de barras de construcción. Entre los factores analizados tenemos:

- Composición química y microestructura

- Propiedades mecánicas y fluencias objetivo

- Caracterización superficial y su efecto en la fractura del material.
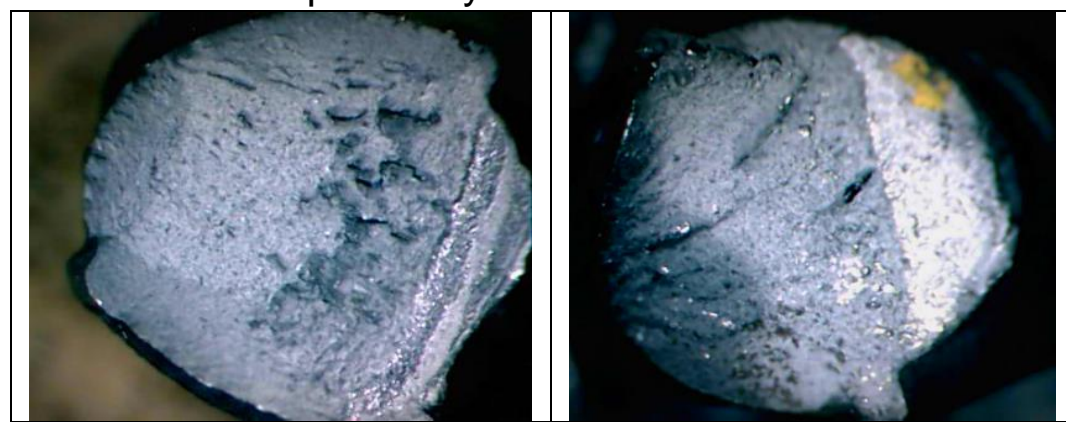

Caso 1

Caso 2
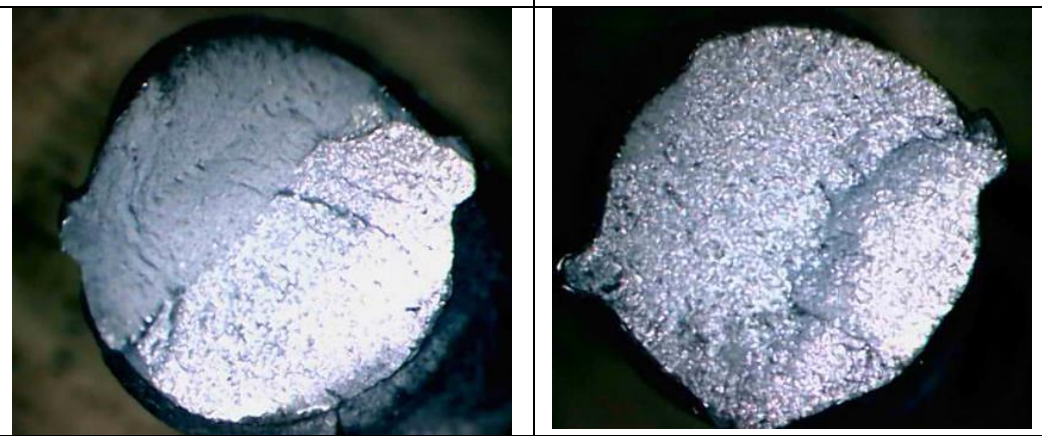

Caso 3

Caso 4

Figura 3. Apariencia de la fractura dúctil (Caso 1) hasta llegar a una fractura frágil (Caso 4)

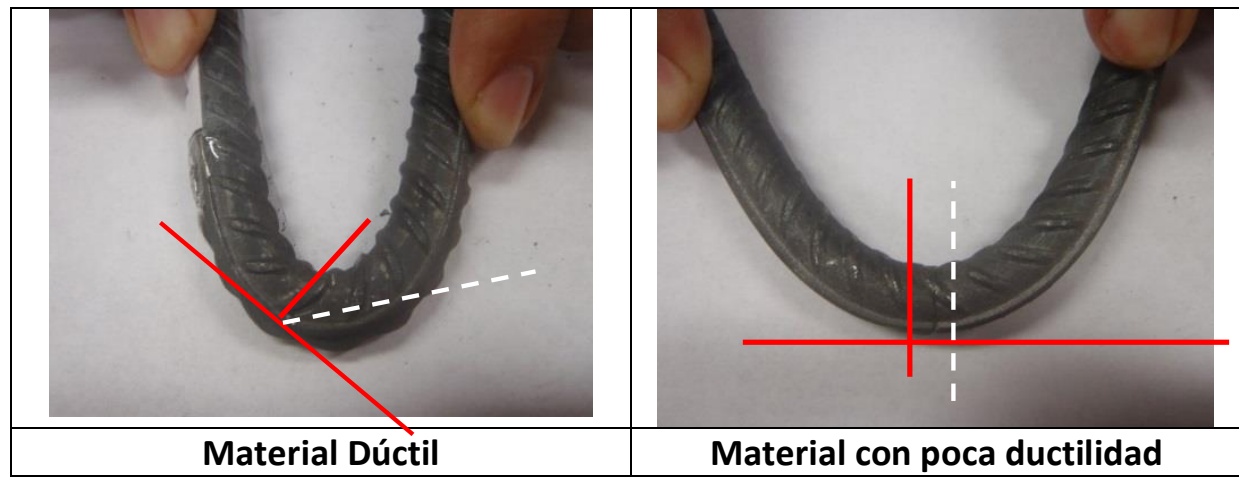

Figura.4 Angulo de rotura de un material dúctil y frágil 


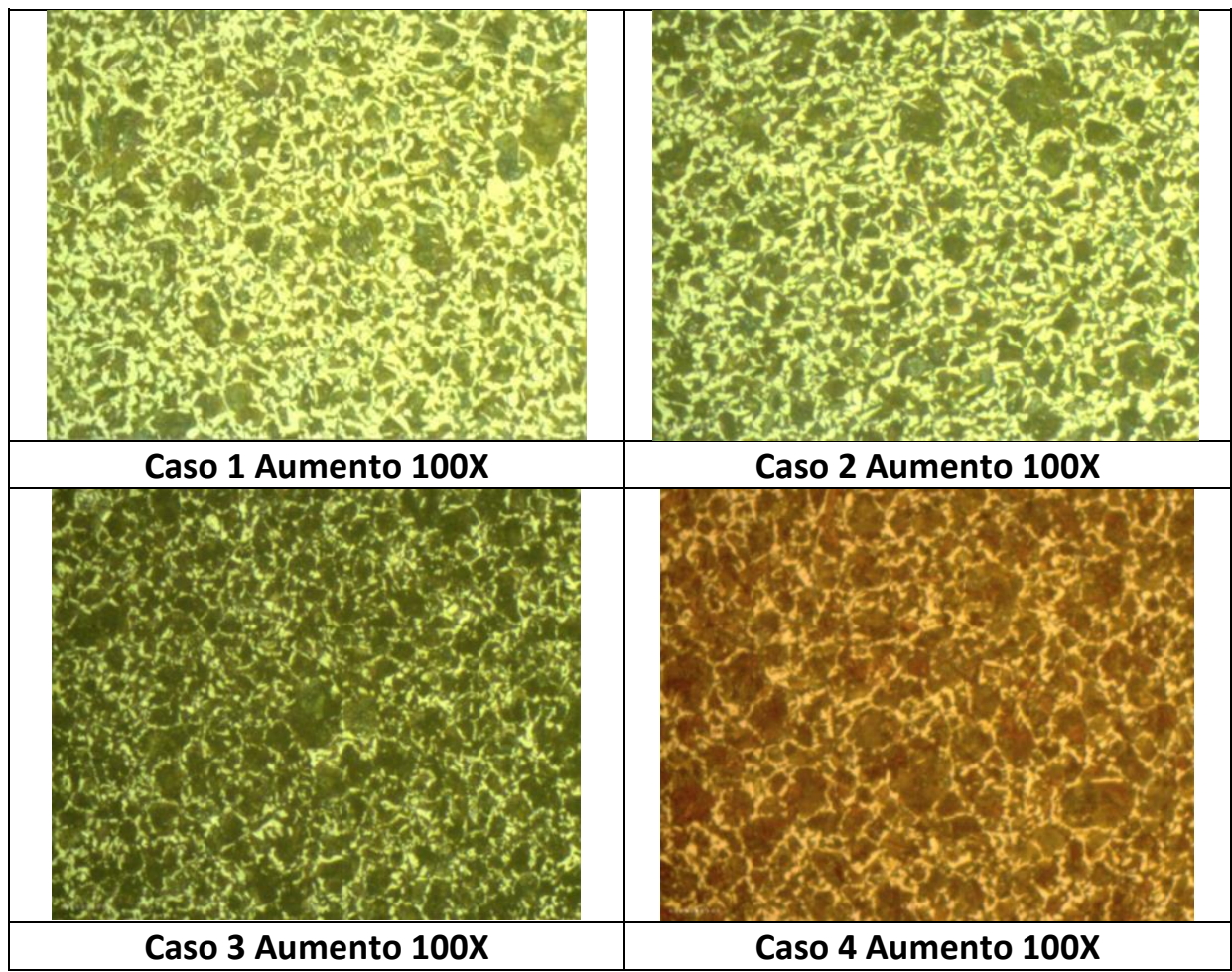

Figura 5. Microestructura de las muestras provenientes de la Figura.3

\subsection{COMPOSICIÓN QUIMICA Y MICROESTRUCTURA}

Elementos residuales tales como $\mathrm{Cr}, \mathrm{Cu}, \mathrm{Sn}, \mathrm{Ni}$, Mo en los aceros al carbono producen efectos adversos en la calidad del producto si no existen medidas preventivas para balancear sus efectos. Actualmente los objetivos en la industria siderúrgica son producir barras de construcción con chatarra de menor costo. Sin embargo, esto ocasiona que los residuales se incrementen y su contenido en el acero varíe de colada a colada el cual hace que las microestructuras varíen al igual que las propiedades mecánicas de fluencia, resistencia a la tracción y alargamiento, produciendo inconsistencia en la tenacidad del producto que se verá reflejado en los ensayos de doblado.

La Figura 6 muestra las variaciones de los elementos residuales a lo largo de la producción de aceros 1040. Como se observa, los valores de $\mathrm{Cr}$, Cu, Sn llegan a picos de 0,25, 0,48 y 0,082 respectivamente, produciendo un acero con mayor porcentaje de constituyentes fragilizantes. El efecto de los elementos estabilizadores ferríticos (p.ej. Cr, Mo, Si) y austeniticos (p.ej. Mn, Ni, Cu, N) ${ }^{(2)}$ es de modificar la cinética de nucleación o crecimiento de la ferrita, perlita o bainita ${ }^{(3)}$ retardando la velocidad de descomposición de la austenita controlada por el proceso de difusión de los residuales al particionar en la interface $\gamma / \alpha$ y $\gamma /$ cementita.

Por tanto, los altos contenidos de elementos residuales producirán un aumento del porcentaje de perlita comparado con un acero de menor contenido de residuales a igual enfriamiento, resultando aceros con diferentes porcentajes de fase de ferrita así como de estructura de perlita. El aumento o disminución de estos elementos 
residuales necesitan ser balanceados con los ajustes de carbón y manganeso para conseguir consistencia en microestructura.

Por otro lado, los contenidos de nitrógeno muestran picos de 350 ppm sobresaturando el acero para contener a los otros gases, produciendo no solo problemas de sopladuras ${ }^{(4,5)}$ en la palanquilla el cual no solo produce problemas en el tren laminador, como también produce problemas de fisuras en el producto terminado, dependiendo del ratio de reducción ${ }^{(6)}$. El efecto del nitrógeno se da en el incremento de microestructuras frágiles debido a su condición de estabilizador austenítico, sin embargo, no es usado para aumentar templabilidad del acero (7). Adicionalmente a esto su efecto como solución sólida crea inestabilidad del material durante la deformación. La Figura 7 muestra la metalografía de un acero 1040 teniendo el mismo carbón equivalente pero con diferente contenido de nitrógeno. Estas dos muestras presentan similar enfriamiento en el proceso productivo, mostrando que el contenido de la fase ferrita se ve reducida en porcentaje a mayor contenido de nitrógeno.

Realizando comparaciones estadísticas de alargamientos de barras 1040 ADN 10 $\mathrm{mm}$ con valores de fluencia similares, muestra la tendencia que a más contenido de nitrógeno se reduce el alargamiento del acero (Figura 8). Otros aspectos que también juegan un rol importarte, son, el efecto del tamaño y homogeneidad del grano austenítico y el espaciamiento interlamelar de la perlita. Por tanto, adicionalmente a los efectos de la composición química, la obtención de una buena microestructura, también dependerá en conocer la temperatura de recalentamiento óptima conociendo TGC (Grain Coarsening temperature), velocidad de deformación, tiempo de recristalización, temperatura de no recristalización, TAr3 y enfriamiento ${ }^{(8,9)}$.

En base a estas variaciones de contenido de residuales se creó una regresión lineal de ajuste de composición química basado en el contenido de elementos residuales para balancear su efecto fragilizador (Figura 9) y garantizar una buena microestructura. Por otro lado era indispensable reducir el contenido de nitrógeno en el acero, sin embargo esta fórmula cuenta rangos de contingencia de carbón para mayor contenidos de nitrógeno fuera de norma interna. Esta regresión lineal tiene como objetivo ajustar el acero para garantizar propiedades mecánicas y de microestructura. Figura 10 muestra la microestructura del acero basado en los cambios realizados. 


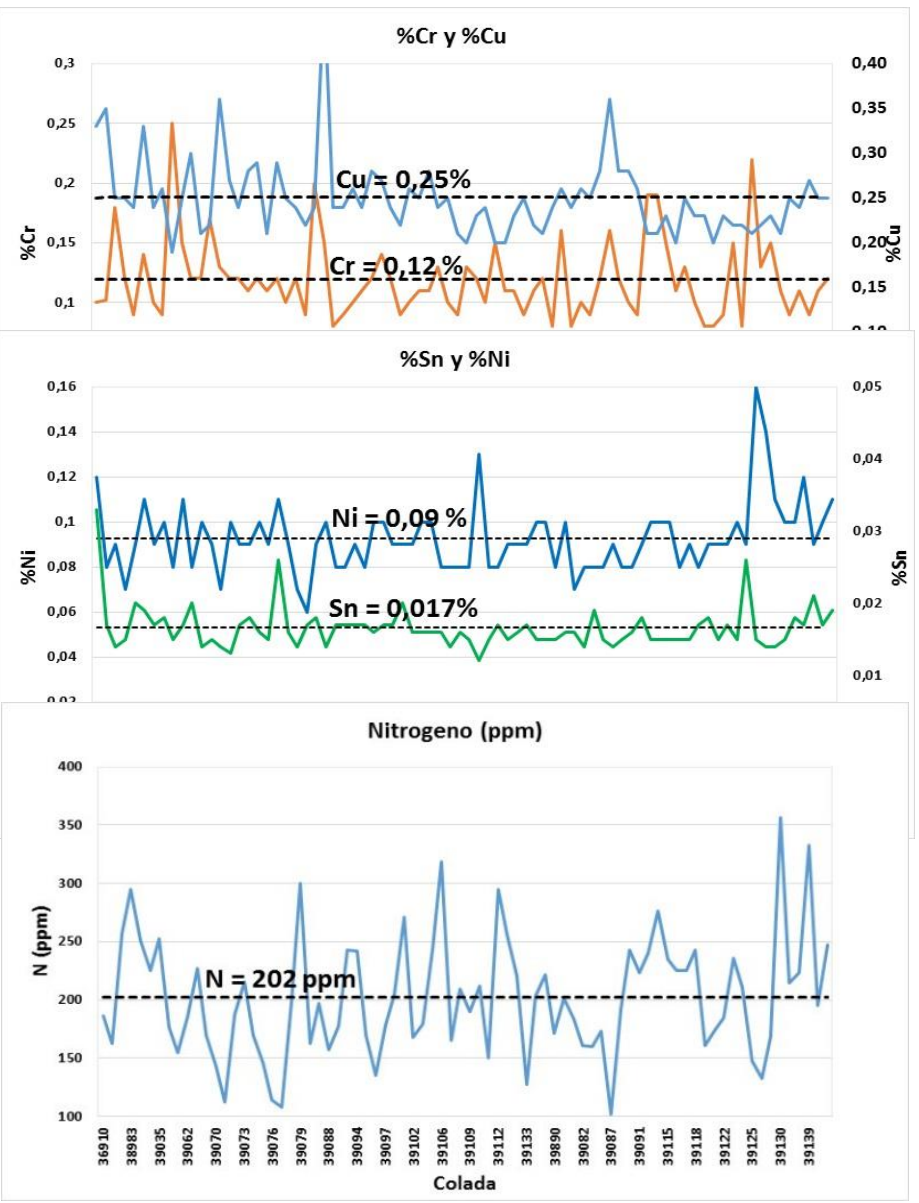

Figura 6. Variación de los elementos residuales y el contenido de nitrógeno final de colada.

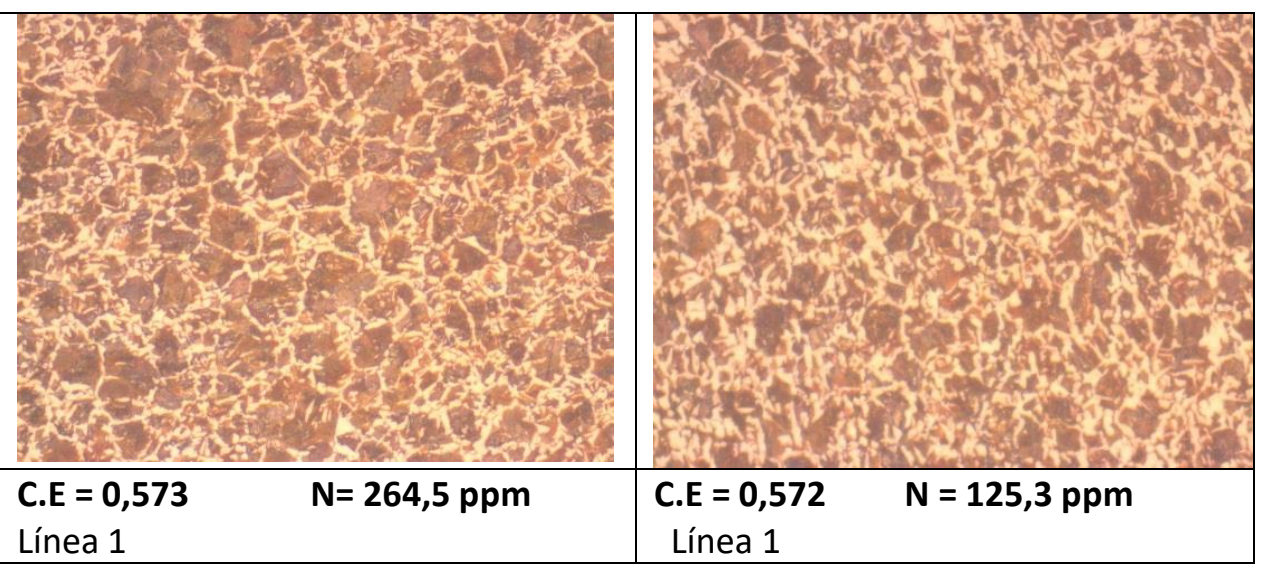

Figura 7. Efecto del nitrógeno para dos aceros con el mismo Ceq, observándose un aumento en la fase ferrítica cuando menor es el contenido de nitrógeno. 


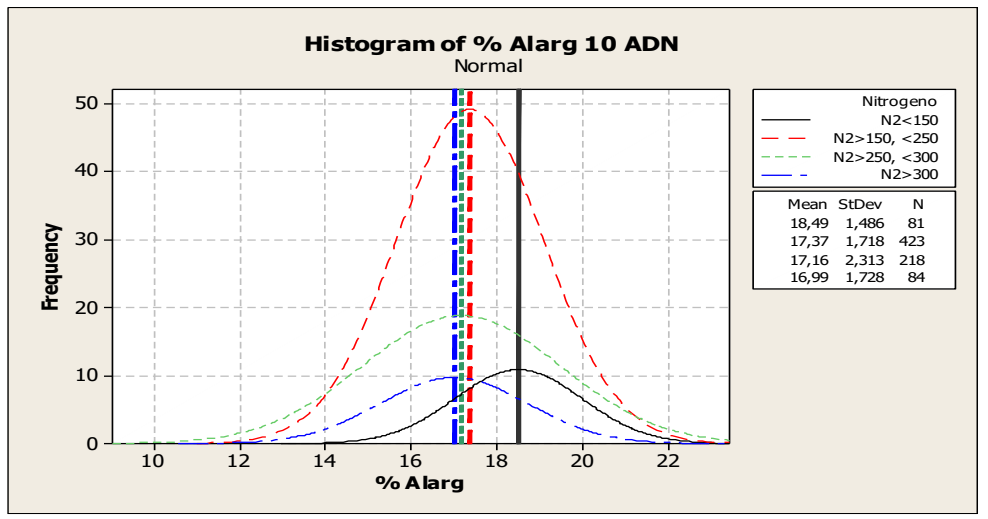

Figura 8. Efecto de tendencia del nitrógeno aceros 1040 ADN 10 mm de una misma fluencia

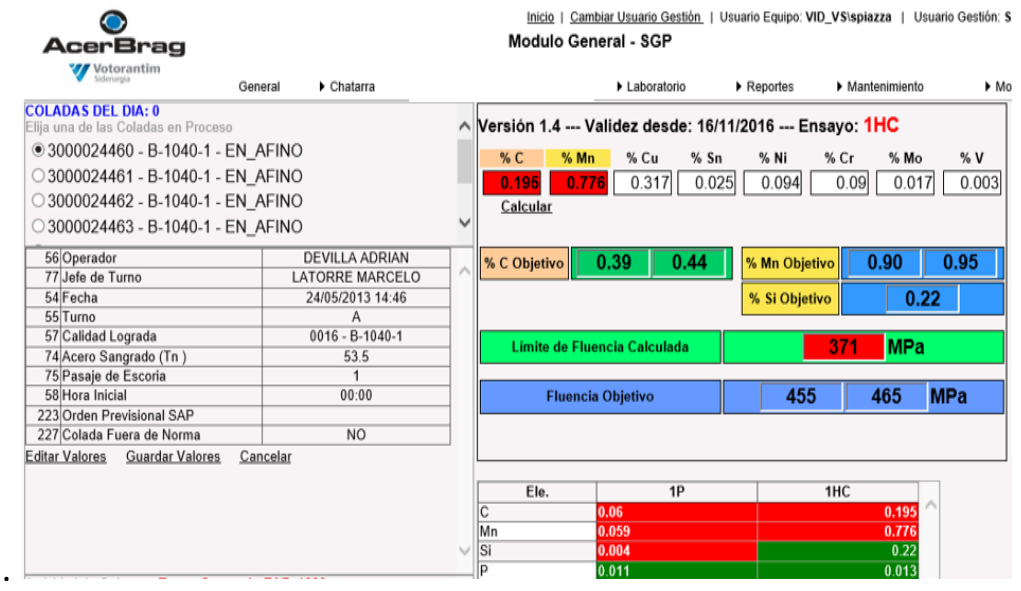

Figura 9. Regresión lineal para ajuste de composición química Acero 1040 ADN 10 mm

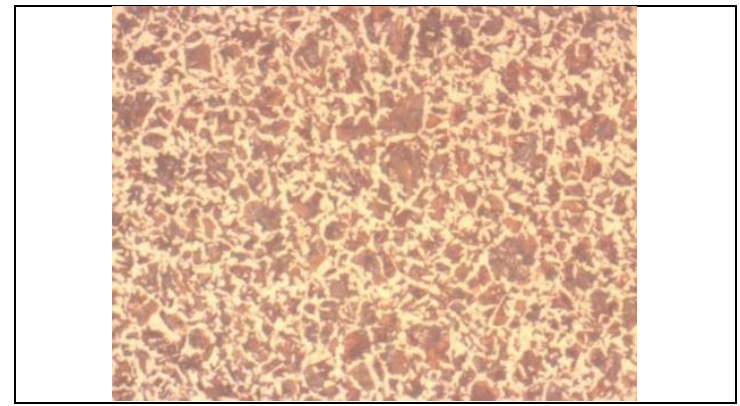

Figura 10. Estructuras del material después de los cambios en composición química

\subsection{PROPIEDADES MECÁNICAS Y FLUENCIAS OBJETIVOS}

Las propiedades mecánicas son establecidas para garantizar que las estructuras metálicas no fallen en servicio. Entre las principales propiedades mecánicas usadas para este producto están, el límite de fluencia, resistencia a la tracción y alargamiento. Aunque los requisitos expresan solo valores mínimos tabla 1, valores elevados de fluencia para estos tipos de acero no cumplirían un doblado exigente. El objetivo es garantizar un rango adecuado que garantice norma y que el material tenga la mejor respuesta en servicio y para ello es importante establecer rangos 
óptimos de fluencia que hagan un material lo más dúctil posible y como vemos en la Figura 11 un rango óptimo de fluencia para garantizar una buena respuesta del material cumpliendo la norma es de 440 - $460 \mathrm{MPa}$.

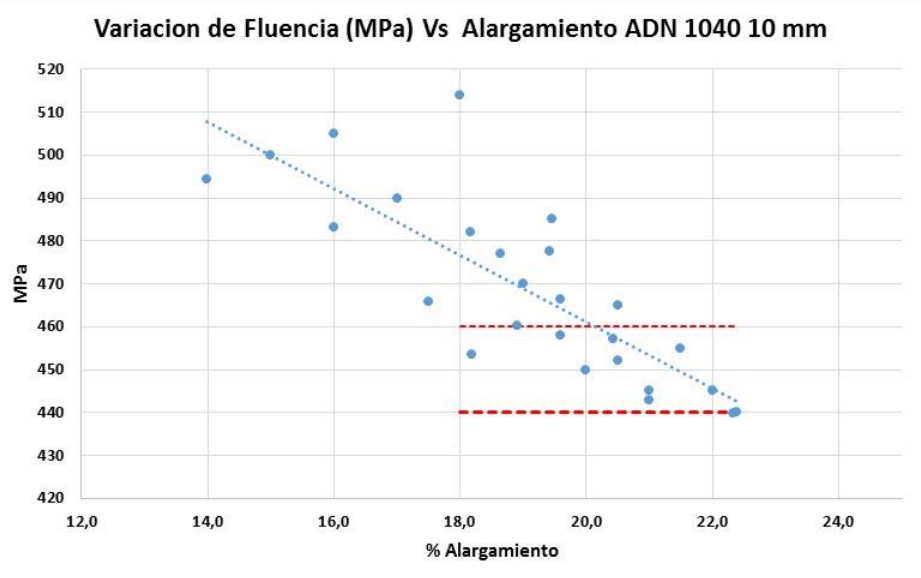

Figura 11. Tendencias del alargamiento con diferentes rangos de fluencia

\subsection{CARACTERIZACIÓN SUPERFICIAL Y SU EFECTO EN LA FRACTURA DEL MATERIAL}

Los factores que gobiernan la velocidad de propagación de un grieta y el colapso final de esta son: la tenacidad del material mostrándose a través del coeficiente de tenacidad de fractura $\mathrm{K}_{\mathrm{IC}}\left(\mathrm{N} / \mathrm{m}^{-3 / 2}\right)$ que es el parámetro de tenacidad o a través del ensayo charpy (Joules). Los dos valores son relacionados en ecuaciones para predecir la vida útil del material ${ }^{(10)}$. La geometría de la fisura y sus dimensiones también juegan un rol importante y finalmente la fuerza aplicada. Estas variables son usadas en Modern Linear Elastic frature mechanics (11).

La Figura 12 muestra el efecto del desgaste de canal creando rugosidades superficiales que se presentan como fisuras en la base del nervio, que aplicando una fuerza como la de doblado genera un campo de tensiones en el vértice de la fisura generando una zona plástica. Si la cantidad de energía por unidad de área es liberada por el material como consecuencia de la rotura de los enlaces atómicos, la fisura se extenderá catastróficamente ${ }^{(12,13)}$. Muchos de estos eventos de rotura por imperfecciones superficiales se han producido en barras de construcción. Para las condiciones de proceso actuales se realizó un modelamiento simples de predicción de la altura del nervio por toneladas producidas (Figura 13) y en combinación con la Figura 14, que representa las alturas de nervio de mecanizado inicial y durante el desgaste que origina problemas de performance del material, se establecieron ventanas de trabajos seguras para producir un tonelaje adecuado de máxima productividad garantizando la calidad del producto. 

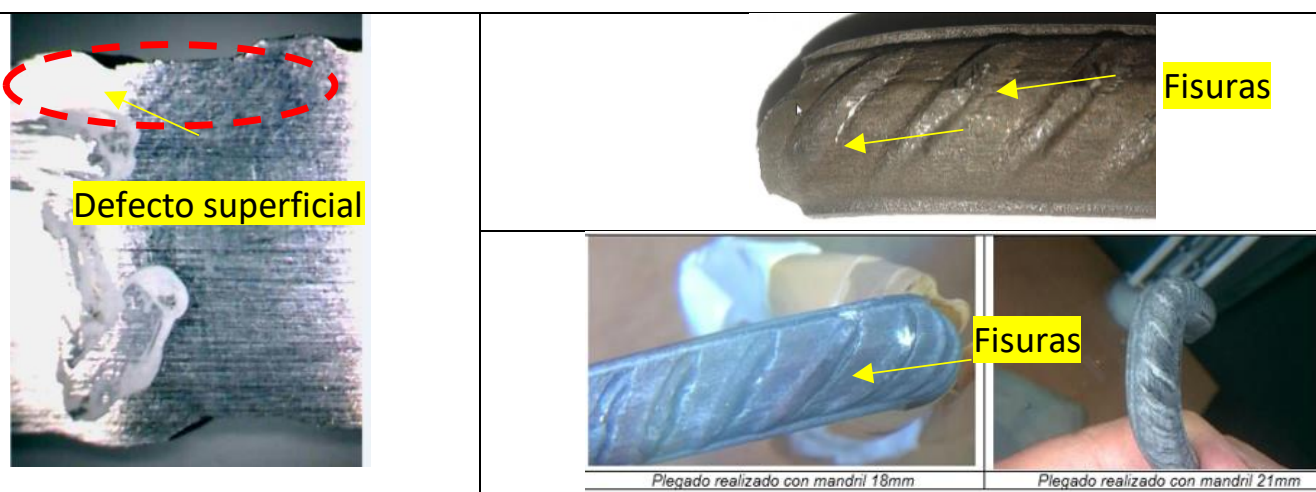

Figura 12. Efecto del desgaste de canal en el doblado de barras.

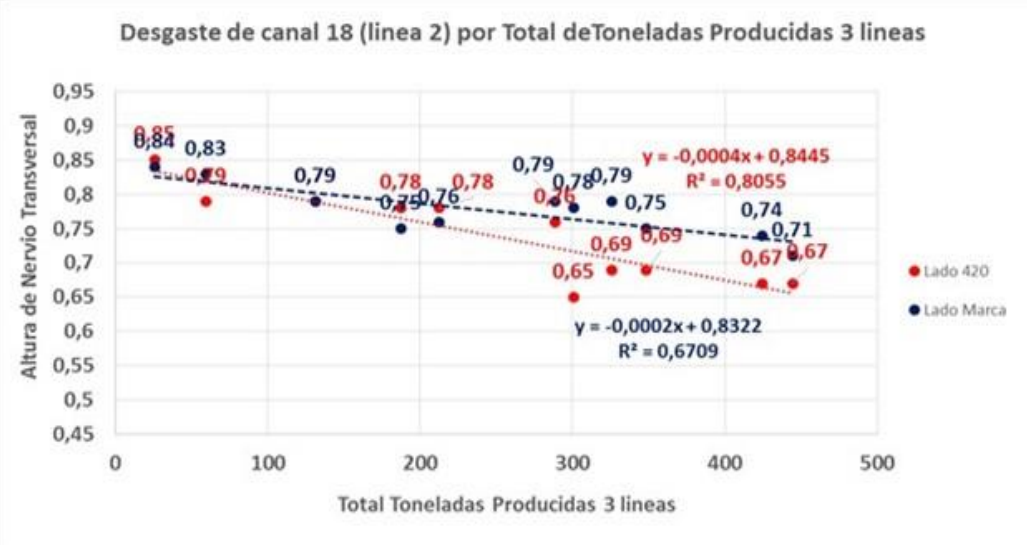

Figura 13. Variación de la altura de canal con el tonelaje producido durante la producción de calidad 1040 ADN

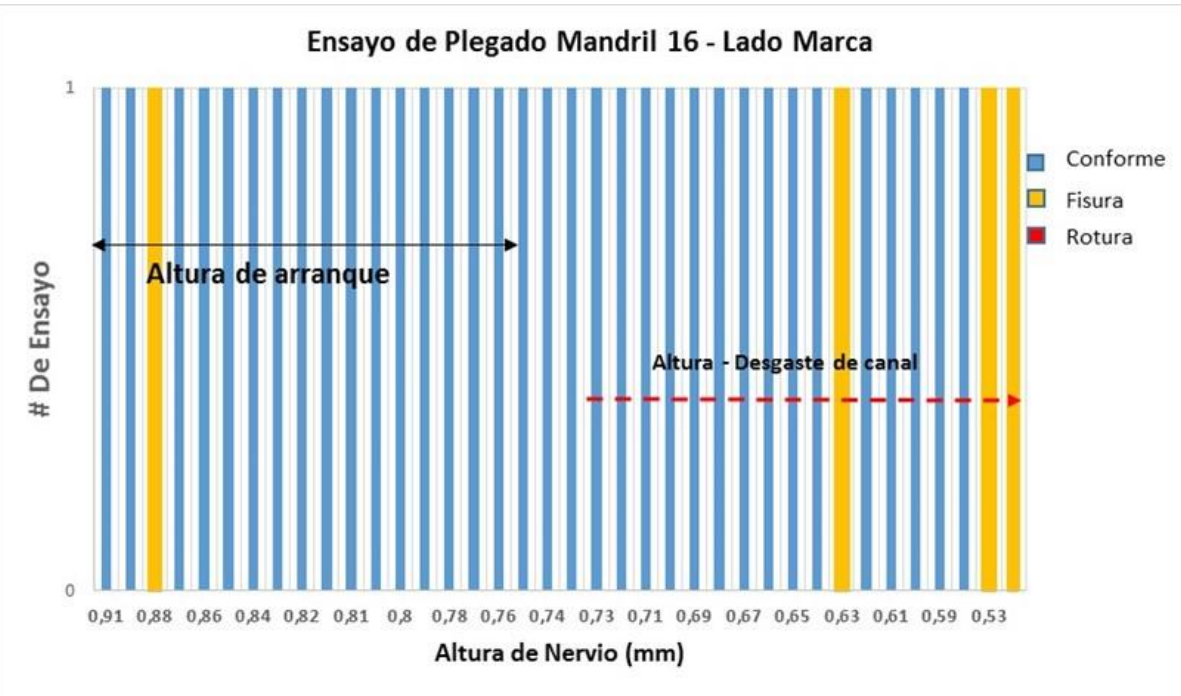

Figura 14. Variación de la altura del nervio inicial y después del desgaste y su efecto en la respuesta al ensayo de plegado, Acero 1040, ADN 10 mm (Mandril $\varnothing 16 \mathrm{~mm}$ ) 


\section{Conclusiones}

- Los métodos de predicción y control son una herramienta importante para establecer condiciones de productividad y calidad del producto. Las contenidos de elementos residuales y su balance con los elementos de ajuste para aceros utilizados en barras nervuradas de medio carbono juegan un papel importante en la calidad de la microestructura del acero. Los métodos de ajuste entre el balance del carbón y manganeso con respecto a los elementos residuales tienen que estar basados con criterios de rango de fluencia, garantizando alargamientos adecuados que logren una buena tenacidad del material. En el presente trabajo se desarrolló modelamientos lineales de predicción basados en fluencias objetivos garantizando buena microestructura y propiedades mecánicas óptimas para el buen desempeño del acero 1040. Por otro lado, también se mostró el efecto nocivo del nitrógeno el cual reduce los alargamientos y crea inestabilidad del material en su respuesta en los ensayos mecánicos.

- La mecánica de fractura de un material contempla condiciones de defectos superficial e interna del material así como el tipo de calidad de acero basados en el Stress intensity factor (KIC) el cual es el reflejo de tenacidad. Se hizo una caracterización del tipo de defecto superficial originado por el desgaste de canal el cual muestra fisuras superficiales que generan campos de tensiones durante los esfuerzos realizados en los ensayos de doblado. El defecto se agrava a medida que el desgaste es más severo creando protuberancias superficiales. Por tanto, la medición indirecta a través de la velocidad de reducción de la altura del nervio y las mediciones de alturas criticas de rotura de material generan una ventana optima de proceso para evitar problemas de rotura por temas de defectos superficiales. El peor escenario mostrado es un acero de baja tenacidad con un microestructura inadecuada y que presenta defectos superficiales internos y externos produciendo una falla prematura del material a esfuerzos exigidos.

- La mejora en la respuesta del material en el doblado para las barras 1040 ADN $10 \mathrm{~mm}$ teniendo en consideración los puntos discutidos anteriormente se muestra en la Figura 15 la cual compara ensayos antes y después de los cambios realizados con un mandril exigido de diámetro $18 \mathrm{~mm}$ (mitad de lo indicado en la norma)

\begin{tabular}{|l|l|l|}
\hline Antes ( Mandril 18) & Ahora ( Mandril 18) \\
\hline & & \\
\hline & & \\
\hline Rotura & $\square$ Grieta & \\
\hline
\end{tabular}

Figura 15. Muestra el cumplimiento de los ensayos de doblado a mandril mitad de norma en los nervios 


\section{AGRADECIMIENTO}

Quiero agradecer a Acerbrag - Votorantim Siderurgia y en especial a los que hicieron posible la realización de este trabajo, al Ing. Juan Ignacio Fortunato, a todo el grupo de calidad y a las áreas productivas por haber permitido realizar las pruebas y estandarizaciones.

\section{REFERENCIAS}

1. Norma IRAM/IAS U-500-528; Barras de acero conformadas de dureza natural para armaduras en estructuras de hormigón, Abril 2017.

2. Professor Eric Palmiere, Lecture note, Physical Metallurgy of steels. University of Sheffield, England 2007.

3. David. A. Porter, Kenneth E. Easterling and Mohamed Y. Sherif, Phase Transformations in Metals and Alloys. Third Edition. 2008. Pag 341

4. Managing the quality of continuously cast steel billet. Lee Fui Tong. School of Engineering. Proceeding of the international conference on recent advances in mechanical \& Material Engineering 30-31 may 2005 Kuala Lumpur, Malaysia Paper 134

5. Ing. Lauro Chevrant, Defecto de palanquillas ,Technical Course held on Aceros Arequipa, 2012.

6. Dr, E.S .Szekers, , Brimacombe Continuous Casting Course. Continuous Casting of steels, Blooms and Slabs, Vancouver - Canada ,May 14 to May 18,2012

7. Dr Alan W. Cramb ,The Making Shaping and Treating of Steel. 11 th Edition Casting Volume. Pag. 219.

8. Professor E.J Palmiere, Lecture Note, Deformation Mechanisms of solids. University of Sheffield, England 2007.

9. C.M sellars, The physical metallurgy of hot working. Processing of an international Conference on hot working and forming Process. The metal Society London. 17-20 July 1979

10. Crack Tolerance Assessment of A Series III Female connector, Prepared for BSW Ballgrab LTD United Kingdom, By Stress Engineering Services, December 2007

11. Dr John Chubb, Prof P.E. Irving ,Crack tip stress field and the local approach to failure prediction. Cranfield University , England. 25 - 29 January 2010

12. Dr D.Cartie , Thermodynamic Criteria for fracture or the work of Alan Arnold Griffith on Brittle Materials (1920) , Cranfield University, England. 25-29 January 2010.

13. Dr Denis Cartie. Failure of Material and Structures (AM FMS), Ductile and Brittle Fracture, Cranfield University, England 25-29 January 2010. 